\title{
Téoros
}

Revue de recherche en tourisme

\section{Réflexions sur la question de la création de la valeur touristique}

\section{Romain Petiot}

Volume 23, numéro 3, automne 2004

URI : https://id.erudit.org/iderudit/1071233ar

DOI : https://doi.org/10.7202/1071233ar

Aller au sommaire du numéro

Éditeur(s)

Université du Québec à Montréal

ISSN

0712-8657 (imprimé)

1923-2705 (numérique)

Découvrir la revue

Citer cet article

Petiot, R. (2004). Réflexions sur la question de la création de la valeur touristique. Téoros, 23(3), 50-54. https://doi.org/10.7202/1071233ar d'utilisation que vous pouvez consulter en ligne.

https://apropos.erudit.org/fr/usagers/politique-dutilisation/ 


\section{Réflexions sur la question de la création de la valeur touristique}

\section{Romain Petiot}

L'énoncé de quelques chiffres permet de donner une bonne idée du poids du secteur touristique dans une économie. En France, par exemple, l'activité touristique a contribué en 2002 à créer 6,7 \% de la richesse nationale ${ }^{1}$. Pour cette même année, les recettes du tourisme international sur le territoire français se sont élevées à 36,6 milliards d'euros. Toujours durant l'année 2002, on a enregistré en France 1,5 milliard de nuitées (Direction du tourisme, 2003). Ces données permettent d'affirmer, à l'instar d'Eadington et Redman (1991: 41), que le secteur touristique est un secteur économique majeur. Il s'agit notamment d'un des secteurs d'activité pour lequel la croissance a été à la fois la plus importante et la plus rapide dans la seconde moitié du vingtième siècle.

L'expansion des activités touristiques trouve naturellement son origine dans la croissance de la demande touristique. Celle-ci s'explique par la conjonction de deux phénomènes liés à la croissance économique mondiale et aux gains généralisés de productivité : d'une part, l'augmentation continue du niveau de vie et du pouvoir d'achat, d'autre part, la croissance du temps libéré des contraintes productives (réduction du temps de travail, allongement de la durée de vie, etc.) (Caccomo et Solonandrasana, 2001 : 9).

Le regard relativement récent de l'économiste sur le secteur touristique révèle toutefois un besoin croissant d'aide à la décision économique, tant de la part du décideur public que du décideur privé. L'analyse économique fournit en effet des outils de compré- hension du comportement des agents économiques nécessaires, in fine, au développement harmonieux de l'activité touristique, c'est-à-dire à l'accroissement de l'efficacité sociale nette du secteur, que ce soit pour les consommateurs (les touristes), les producteurs de l'offre touristique, les décideurs publics ou l'ensemble des agents économiques qui évoluent dans l'environnement de l'activité touristique (les non-touristes).

L'analyse économique reposant sur le concept de valeur, l' analyse économique du tourisme exige une définition rigoureuse du concept de «valeur touristique». Ainsi, après un retour sur la définition de la notion de «produit touristique», le présent article soumet à la discussion un certain nombre de réflexions relatives à la délimitation des concepts de «valeur touristique» et de «création de la valeur touristique». On s'interroge en particulier sur la pertinence du mécanisme de formation de la valeur touristique qui repose traditionnellement sur la base des dépenses touristiques, alors que l'activité touristique recouvre, entre autres, la consommation de biens dont les prix ne sont qu'implicites (temps, ressources naturelles, infrastructures publiques, etc.) ou des activités sources d'externalités ${ }^{2}$.

\section{L'échange du produit touristique crée la valeur touristique}

Le meilleur moyen de cerner le concept de valeur touristique est de revenir sur la définition classique du «tourisme» énoncée par l'Organisation mondiale du tourisme: «le tourisme comprend les activités déployées par les personnes au cours de leurs voyages et de leurs séjours dans des lieux situés en dehors de leur environnement habituel pour une période consécutive qui ne dépasse pas une année, à des fins de loisirs, pour affaires et autres motifs » (OMT, 1994). D'un point de vue économique, cette définition conduit à caractériser l'activité touristique par les «dépenses touristiques». En effet, le tourisme est défini par les activités déployées par les touristes. Il n'existe donc pas de produit par essence touristique. Un produit, c'est-à-dire un bien ou un service, devient un produit touristique à partir du moment où le consommateur de ce produit est identifié comme touriste (Sessa, 1989; Cuvelier, 1998; Caccomo et Solonandrasana, 2001). Il en découle naturellement que ce sont les dépenses des consommateurs réputés touristes qui sont à l'origine de la valeur touristique. Dès lors, le prix observé lors de l'échange d'un produit entre un touriste et l'industrie touristique constitue l'élément de base permettant de mesurer la valeur touristique.

De cette définition, il est possible de délimiter le champ de l'analyse économique appliquée au secteur touristique. D'une part, le produit ne prenant sa qualité de produit touristique qu'à partir du moment où il est consommé par un touriste, l'analyse de la demande constitue le point de départ naturel de l'analyse économique du tourisme (Stafford, 1996). L'analyse économique de la demande touristique se propose ainsi d'explorer l'expression du goût des consommateurs en matière de tourisme dans le but de fournir une description de la demande de produits touristiques. On recense sur ce point nombre d'écrits (Morley, 1991 ; Papatheodorou, 2001 ; Randriamboarison, 2003). D'autre part, la valeur touristique résultant de la transaction d'un 


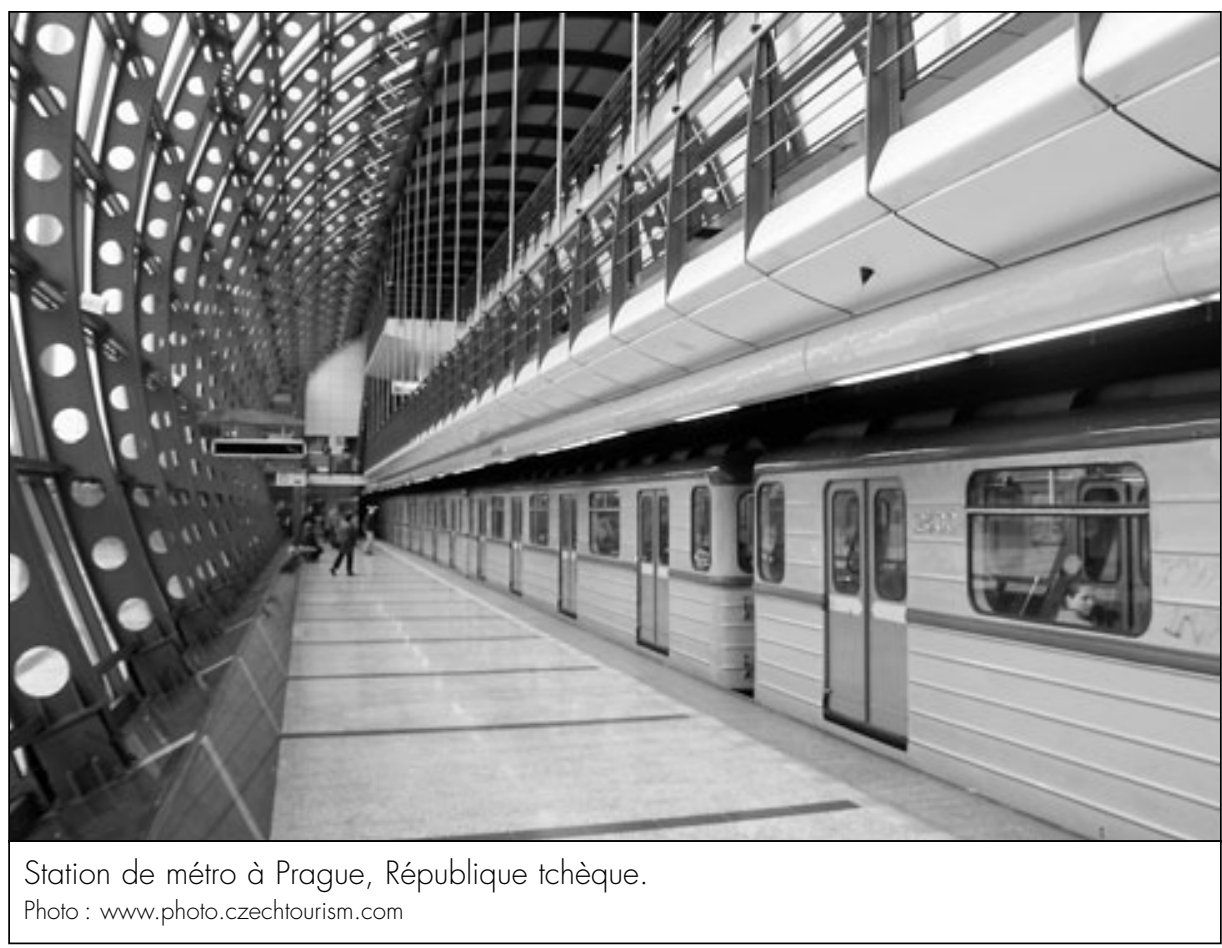

produit entre le touriste et l'industrie touristique, outre l'analyse de la demande, l'analyse économique du tourisme aborde également l'offre de produits touristiques. L'analyse de l'offre touristique se traduit par une documentation qui traite de l'organisation de l'industrie du tourisme et discute des conditions d'expression de la concurrence au sein du secteur touristique (Hodgson, 1987 ; Poon, 1993 ; Brillard, 2000 ; Caccomo et Solonandrasana, 2001).

Pour résumer, le concept de valeur touristique s'élabore sur la base de la définition du produit touristique : c'est l'échange de produits entre des touristes et l'industrie touristique qui crée la valeur touristique. Elle se mesure par le prix constaté lors de l'échange. Les dépenses touristiques permettent donc d'évaluer l'activité touristique. Cependant, se contenter du seul prix du produit touristique pour valoriser l'activité touristique conduit, d'un point de vue économique, à limiter stricto sensu le champ de l'analyse aux transactions entre les touristes et l'industrie touristique. Or, si la transaction semble être un argument pertinent de mesure de la valeur touristique, il existe d'autres lieux de création de la valeur touristique. En effet, l'exercice de l'activité touristique nécessite l'usage d'infrastructures, notamment d'infrastructures

publiques, dont la valeur n'apparaît pas, du moins en première approche, dans les transactions effectuées entre les touristes et l'industrie touristique. En outre, l'activité touristique a un effet sur les activités non touristiques de son environnement. A priori, cet effet n'est pas valorisé dans les transactions entre les touristes et l'industrie touristique, surtout lorsqu'il n'a pas de réalité monétaire. Il semble dès lors cohérent de se poser la question de savoir si le prix du produit touristique rend effectivement compte de l'intégralité de la valeur créée par l'activité touristique.

\section{Le prix du produit touristique rend-il parfaitement compte de la valeur touristique?}

Le prix du produit touristique intègre-t-il
les dépenses publiques nécessaires à
l'exercice des activités touristiques?

Un prolongement de la définition du produit touristique conduit à s'interroger sur la pertinence de mesurer précisément la création de valeur touristique par les dépenses touristiques. Le produit touristique est en effet un «produit complexe » (Caccomo et Solonandrasana, 2001 : 17). En l'occurrence, le produit touristique peut être défini comme un «continuum de biens privés, biens publics et biens publics locaux » (Brillard, 2000 : 47). Par exemple, un produit touristique peut certes contenir un voyage et des activités annexes vendus par un tour-opérateur, mais il englobe aussi les infrastructures nécessaires à la réalisation de ce voyage et de ces activités annexes. Dans cet exemple, on peut légitimement s'interroger sur le fait que le prix auquel le produit est échangé entre un touriste et le tour-opérateur contienne la valeur des dépenses publiques engagées dans la production de biens publics pourtant indispensables à l'échange du produit touristique. La question s'avère plus redoutable encore lorsqu'il s'agit de considérer les dépenses publiques engagées pour la fourniture de biens publics non marchands. Par définition, en effet, les biens publics non marchands n'ont pas de prix dans la mesure où ils ne font pas l'objet d'un échange sur un marché. Par conséquent, si l'on considère que le produit touristique est un produit complexe et si, de plus, on envisage que le produit touristique contient des biens publics, notamment des bien publics non marchands, la question de savoir si le prix du produit touristique rend bien compte de l'intégralité de la valeur touristique, notamment si les dépenses touristiques incorporent les dépenses publiques nécessaires au financement et à l'exploitation des biens et services publics indispensables à l'exercice de l'activité touristique, prend tout son sens.

La réponse semble être négative lorsqu'on s'en tient à la stricte définition du produit touristique. En effet, le produit touristique se limitant strictement au produit échangé entre le touriste et l'industrie touristique, il n'y a pas de raison que le prix auquel est échangé ce produit contienne la valeur des biens et services publics produits pour l'exercice de l'activité touristique, a fortiori la valeur des services non marchands. Deux exemples permettent néanmoins de nuancer cette conclusion.

La première illustration renvoie aux fonds publics que les collectivités territoriales en charge de la gestion des espaces touristiques engagent pour collecter et traiter les déchets, notamment les eaux usées. 
Selon le Réseau national de données sur l'eau (RDNE), les agglomérations touristiques françaises de plus de 10000 habitants sont responsables d'environ $8 \%$ de la pollution brute des eaux (Institut Français de l'Environnement, 2000). Il existe donc un besoin réel de financement public du traitement des eaux usées spécifiquement rattaché à l'exercice des activités touristiques. En termes économiques, ce financement public contribue à créer une valeur directement liée à l'activité touristique. Il semble néanmoins que le prix des produits touristiques ne considère pas directement ces dépenses. On peut raisonnablement penser cependant, en se référant au Code général des collectivités territoriales, que les sommes prélevées via la taxe de séjour permettent de financer ces dépenses publiques ${ }^{3}$. Au demeurant, si la taxe de séjour n'apparaît pas clairement dans les Comptes du tourisme, ces dépenses se retrouvent dans les dépenses touristiques recensées traditionnellement par la statistique publique ${ }^{4}$. Ce premier exemple tend à montrer que si le prix des produits touristiques ne rend pas directement compte des dépenses publiques indispensables à l'exercice de l'activité touristique, elles apparaissent cependant bien dans l'échange des produits touristiques et concourent à la création de la valeur touristique.

La deuxième illustration porte sur les dépenses publiques nécessaires au financement et à l'exploitation des infrastructures routières gratuites. À nouveau, ces dépenses n'apparaissent pas directement dans l'échange du produit touristique. Pourtant, sans investissement dans de telles infrastructures, l'activité touristique ne pourrait pas ou, à tout le moins, pourrait mal s'exercer'. L'usage de l'infrastructure routière gratuite pour des motifs touristiques est une consommation sans contrepartie monétaire, mais qui reflète une véritable création de valeur. Par convention, cette valeur d'usage est estimée par les dépenses publiques engagées dans le financement de l'infrastructure. Si ces dépenses ne sont pas explicitement contenues dans le prix observé lors des transactions du produit touristique entre les touristes et l'industrie du tourisme, il semble néanmoins évident que le prix des

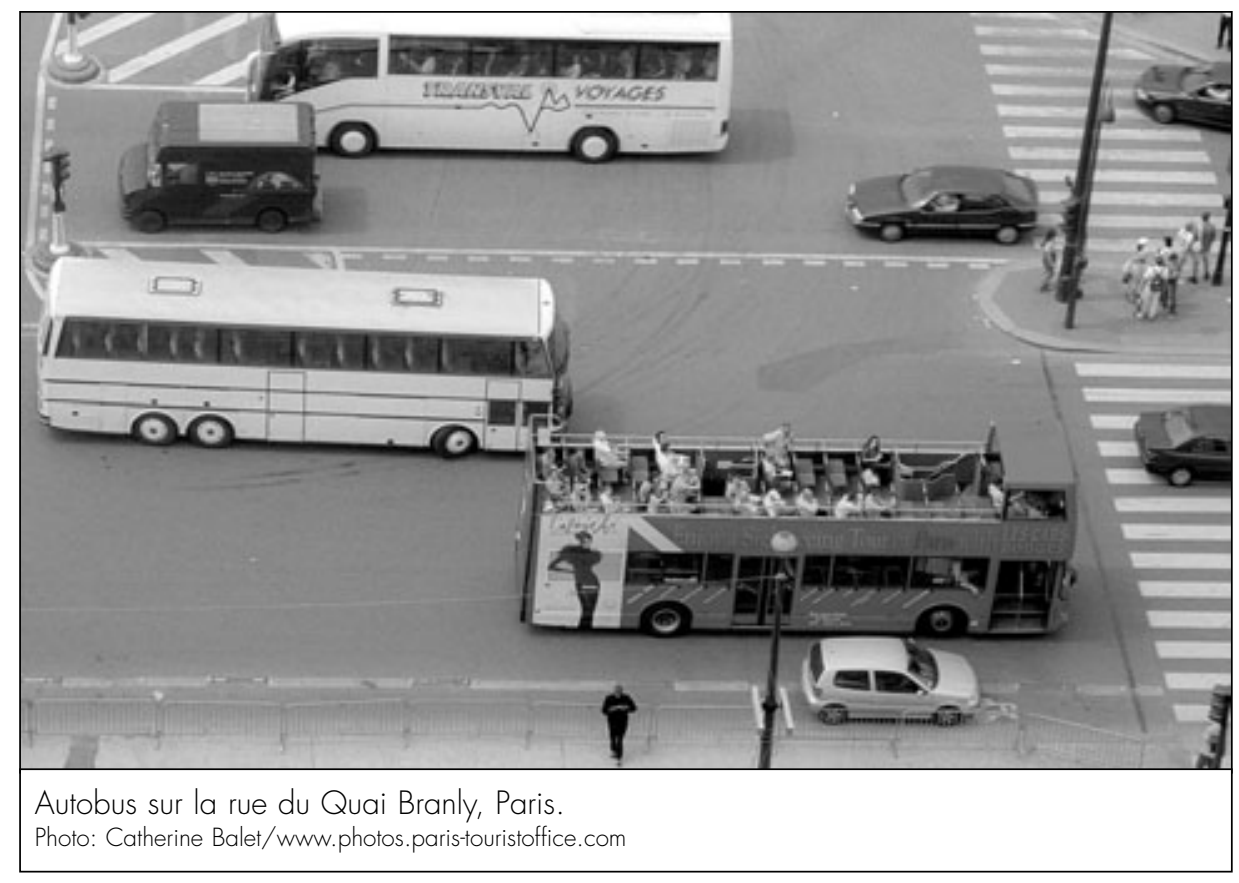

produits touristiques soit sensible à la présence d'infrastructures routières. L'existence d'une telle infrastructure a, par exemple, un effet réel sur le prix des biens immobiliers, que ce soit à la baisse, en cas de nuisances créées par l'infrastructure, ou que ce soit à la hausse, lorsque l'infrastructure améliore sensiblement l'accessibilité des espaces touristiques. Le prix des produits touristiques capitalise en quelque sorte les dépenses publiques nécessaires au financement de ce type d'infrastructures. Dès lors, par un simple mécanisme de capitalisation foncière, le fonctionnement décentralisé du marché, qui conduit à la formation du prix du produit touristique, semble bien intégrer la création de valeur touristique issue de ces dépenses publiques.

En première analyse, il semble que le prix du produit touristique mesure relativement bien la création de valeur touristique. Non seulement il relate parfaitement la valeur créée dans l'échange du produit touristique, mais il intègre également la valeur créée par les dépenses publiques nécessaires à l'échange du produit touristique. Le prix d'échange d'un produit entre un touriste et l'industrie touristique est donc un indicateur de bonne qualité de la création de la valeur touristique, même lorsqu'une partie de cette valeur touristique échappe à la sphère marchande. Les dépenses touristiques constituent par conséquent une bonne mesure de la valeur touristique. On peut cependant s'interroger sur l'aptitude du prix du produit touristique à intégrer d'autres types de biens publics comme les effets externes qui, selon qu'ils sont positifs ou négatifs, participent également à la création de la valeur touristique.

Le prix du produit touristique tient-il compte des externalités produites par l'activité touristique?

L'économiste parle d'effet externe, ou d'externalité, lorsque l'activité d'un agent économique (consommateur ou producteur) a un effet sur la fonction-objectif d'un autre agent économique sans que cet effet fasse l'objet d'une contrepartie monétaire. Un effet externe a donc une réalité en termes de variation du niveau d'utilité pour un consommateur ou du niveau de profit pour un producteur, mais il n'a aucune réalité monétaire, donc aucun prix (Laffont, 1988: 13). Lorsque l'effet externe produit par un ou plusieurs agents économiques influe sur la fonction-objectif de l'ensemble des autres agents économiques présents sur le marché, on peut alors être conduit à considérer l'effet externe comme un bien public (Greffe, 1994: 126). Lors de l'échange de produits entre les touristes et l'industrie touris- 
tique, il se peut que des externalités se produisent au sein de la sphère touristique, mais également sur l'ensemble des agents économiques. La question qu'il convient par conséquent de poser est de savoir si le prix du produit touristique rend compte des effets non monétaires que l'activité touristique peut générer sur l'activité économique des agents qui évoluent dans son environnement, y compris sur l'activité des non-touristes. Deux exemples permettent à nouveau d'illustrer le problème.

Le premier exemple concerne la pollution atmosphérique. Il existe sur ce point deux niveaux à considérer : une pollution locale et une pollution globale. La concentration de polluants locaux sur un site (effluents chimiques tels que les particules - PM - les oxydes d'azote $-\mathrm{NO}_{\mathrm{X}}$ - et autres composés organiques volatiles - COV), souvent liée au dynamisme de l'activité touristique, a un effet direct sur l'attrait que présente ce site, donc sur l'étendue future de l'activité touristique. La pollution dite locale contribue, d'une part, à dégrader l'offre touristique en qualité et, d'autre part, à altérer la satisfaction que peut procurer la consommation de produits touristiques localisés sur ce site. Cette dégradation a un effet sur le prix des produits touristiques, notamment sur les prix fonciers, la pollution conduisant à déplacer la demande touristique vers des sites où la pollution est moindre. Dès lors, lorsqu'il s'agit de pollution atmosphérique locale, le prix de marché donne une parfaite idée de son effet sur la valeur touristique. L'exemple peut du reste être étendu à d'autres types de nuisances localement concentrées, comme le bruit ou la pollution des eaux. Il peut également comprendre d'autres types d'effets comme, entre autres, les effets structurants qu'un site touristique peut avoir pour l'attractivité d'autres sites touristiques ${ }^{6}$ ou pour la réputation d'accueil d'une région, par exemple. L'attrait d'un site peut donc contribuer à l'augmentation des dépenses dans d'autres sites, ce qui constitue au demeurant un effet directement intégré dans la sphère marchande. En revanche, la question de la prise en compte des effets de la pollution par les prix touristiques devient beaucoup plus complexe lorsqu'il s'agit d'intégrer la pollution dite globale (les gaz à effet de serre comme principalement le dioxyde de carbone - $\mathrm{CO}_{2}$ - produit essentiellement par les transports). L'activité touristique contribue en effet à la création d'une partie de la pollution globale. Si l'état des connaissances en la matière permet raisonnablement d'estimer une partie de la production de la pollution globale directement imputable à l'activité touristique (voir le tableau 1), il n'en demeure pas moins que cette mesure n'a aucune traduction monétaire dans la création de la valeur touristique, en particulier lorsque cette valeur est fondée sur les dépenses touristiques. Comment intégrer dans le prix d'un produit touristique situé en Espagne, dépensé à l'année $n$, les dépenses publiques et privées engagées à l'année $n+m$ liées à la dégradation de la santé d'agents économiques vivant en France ou au Mexique? La question reste largement ouverte, mais il paraît évident que l'activité touristique ayant une part non négligeable dans la production de la pollution atmosphérique globale, il faudra bien lui trouver des réponses.

La deuxième illustration porte sur la question de la congestion routière. L'exercice de l'activité touristique conduit le touriste à se déplacer, soit pour rejoindre son lieu de villégiature, soit pour rayonner aux environs de ce lieu de villégiature. Quel que soit son mode de transport, le touriste participe à la création, selon une saisonnalité marquée, d'une demande de transport qui vient s'ajouter à la demande locale de déplacements; ponctuellement, elle conduit à des situations de congestion à l'origine de pertes de temps de transport considérables, à la fois pour la population touristique et pour la population des nontouristes, qu'elle soit locale ou en transit. Il y a là un risque de confrontation, sur une même offre de transport, de demandes de transport aux motifs non seulement différents, mais presque antagonistes (travail versus loisirs), sources en outre d'insécurité. Les valeurs du temps perdu par les usagers non-touristes sont-elles prises en compte dans la valeur touristique ? Il semble qu'il soit difficile de trouver dans les dépenses touristiques une quelconque trace de la dégradation de leur satisfaction, en tout cas lorsque ce phénomène se déroule sur des infrastructures gratuites. Pourtant, un certain nombre d'agents économiques voient se détériorer leur satisfaction sans en retirer de compensation monétaire. L'exemple montre en quoi il peut s'avérer fructueux de réfléchir à la notion de création de valeur touristique, non plus en se limitant à la relation touristes / industrie touristique, mais en ouvrant le champ d'analyse à la relation touristes / industrie touristique / non-touristes.

On pourrait bien entendu développer d'autres exemples d'effets externes, tant positifs que négatifs, qui conduisent à se poser la question de la pertinence du seul prix du produit touristique pour évaluer la création de valeur touristique. Par exemple, la surexploitation d'un site peut avoir des effets négatifs sur la qualité même du site ${ }^{7}$, voire sur la qualité et l'identité d'autres sites ${ }^{8}$ ou sur la qualité de vie quotidienne de la population locale (sentiment d'envahissement, inflation importée, etc.).

\begin{tabular}{|l|c|c|c|}
\hline \multicolumn{4}{|c|}{ TABLEAU 1 : Émissions de dioxyde de carbone $\left(\mathrm{CO}_{2}\right)$ liées aux déplacements touristiques } \\
en véhicules particuliers (VP) des résidants en France en 1994
\end{tabular}

Source: Institut Français de l'Environnement (2000 : 55). 
Au final, se pose la question de la création de valeur liée à la production d'externalités positives ou négatives de la part de l'activité touristique. La valorisation par le marché fonctionne en ce qui concerne les effets localisés et internes à la sphère touristique. La question est davantage complexe lorsqu'il s'agit d'aborder des situations pour lesquelles le marché ne parvient pas seul à internaliser ces externalités (cas de la pollution atmosphérique globale ou de la congestion routière). Il convient tout d'abord de s'interroger sur les outils de mesure de ces externalités et d'en délimiter le concernement. Il s'agit ensuite de réfléchir sur les mécanismes qui conduisent à leur valorisation. Il faut, pour finir, envisager des méthodes d'internalisation qui permettent au marché d'intégrer ces phénomènes économiques par le biais des dépenses touristiques.

\section{Conclusion}

Cette discussion tend à montrer que le prix observé lors de la transaction d'un produit touristique donne un aperçu relativement correct de la valeur touristique. En se fondant sur la définition classique de l'activité touristique, les dépenses touristiques offrent un bon indicateur de la création de la valeur touristique, suffisamment pertinent pour qu'il prenne en compte une grande partie de la valeur créée dans l'activité touristique, y compris celle qui échappe initialement au marché. Néanmoins, lorsqu'on envisage d'ouvrir le champ d'analyse, soit d'intégrer les conséquences de l'activité touristique sur la sphère non touristique, par nature exclues de l'analyse dans la mesure où le mécanisme de valorisation touristique se limite à la définition classique du tourisme, les dépenses touristiques ne suffisent plus. Le prix du produit touristique omet en effet de considérer un certain nombre d'éléments qui contribuent pourtant à faire plus ou moins varier la valeur touristique. L'analyse économique du tourisme gagne alors à recourir aux outils de l'économie publique qui lui permettent d'intégrer une partie de la complexité du produit touristique.

\section{Romain Petiot est maître de conféren-} ces en sciences économiques à l'Université de Perpignan (France).
Notes

1 À titre de comparaison, en 2002, la branche transports a réalisé en France 3,9\% du produit intérieur brut (DAEI, SES, 2003).

2 Le lecteur doit noter que le propos de cet article n'est pas de s'intéresser à la question de savoir si la création de valeur touristique est un vecteur de développement, notamment pour les populations locales. Il est en revanche évident que, une fois le concept de création de valeur touristique parfaitement cerné, il deviendra nécessaire de réfléchir au lien entre croissance économique créée par les activités touristiques et développement, afin, le cas échéant, de construire une batterie d'indicateurs pertinents qui permettront d'identifier le rôle réel du tourisme dans le développement.

3 En France, l'article L 2333-27 du Code Général des Collectivités Territoriales précise que «le produit de la taxe de séjour [...] est affecté aux dépenses destinées à favoriser la fréquentation touristique de la commune. [...] le produit de la taxe peut être affecté [...] aux dépenses destinées à favoriser la protection et la gestion de leurs espaces naturels à des fins touristiques».

\section{Bibliographie}

Brillard, Delphine (2000), Analyse des prix et des caractéristiques de l'offre touristique: Une application aux stations de sports d'hiver françaises, thèse de doctorat de sciences économiques, Université Pierre Mendès, Grenoble.

Caccomo, Jean-Louis, et Bernardin Solonandrasana (2001), L'innovation dans l'industrie touristique : enjeux et stratégies, Paris, L'Harmattan.

Cuvelier, Pierre (1998), Anciennes et nouvelles formes de tourisme. Une approche socio-économique, Paris, L'Harmattan.

Direction du tourisme (2003), Comptes satellites du tourisme, Paris, Ministère de l'Équipement, des Transports, du Logement, du Tourisme et de la Mer.

Eadington, William R., et Milton Redman (1991), «Economics and Tourism», Annals of Tourism Research, vol. 18, p. 41-56.

Greffe, Xavier (1994), Économie des politiques publiques, Paris, Dalloz.

Hodgson, Adèle (1987), The Travel and Tourism Industry. Strategy for the Future, Oxford, Pergamon Press.

Institut Français de l'Environnement (IFEN) (2000), Les indicateurs Tourisme Environnement Territoires, Orléans.

Laffont, Jean-Jacques (1988), Fondement de l'économie publique, Paris, Économica.
4 L'agrégat «T2 - consommation touristique intérieure » des Comptes du tourisme comprend en effet «les dépenses des pouvoirs publics et des administrations en charge du tourisme» qui s'élèvent à 2,33 milliards d'euros en France en 2002 (Direction du tourisme, 2003).

5 L'activité touristique ne justifie pas à elle seule, ou que très rarement, l'existence de ce type d'infrastructures. En revanche, elle en explique souvent le dimensionnement.

6 Le pèlerinage de Lourdes (Hautes-Pyrénées, France) a, par exemple, permis le développement de l'exploitation touristique d'un réseau dense de grottes dans les Pyrénées avoisinantes.

7 L'exploitation intensive de la Grotte de Lascaux (Dordogne, France) est à l'origine de lourdes dégradations qui ont conduit les autorités publiques à fermer le site en 1963. L'exploitation touristique n'a pu reprendre qu'en 1983 avec l'ouverture d'un fac-similé du site, Lascaux II.

8 La surexploitation du tourisme d'achat en Principauté d'Andorre dégrade non seulement le site naturel montagneux (pollution atmosphérique, sonore ou encore visuelle), mais également l'image de la Principauté qui mène régulièrement des campagnes de promotion des autres attraits touristiques du site.
Ministère de l'Équipement, des Transports, de l'Aménagement du territoire, du Tourisme et de la Mer, Direction des Affaires Économiques et Internationales, Service Économique et Statistiques (DAEI, SES) (2003), Les comptes des transports en 2002, Paris.

Morley, Clive L. (1991), « Modeling International Tourism Demand: Model Specification and Structure », Journal of Travel Research, vol. 30, $\mathrm{n}^{\circ} 1$, p. 40-44.

OMT (Organisation mondiale du tourisme) (1994), Recommandations sur les statistiques du tourisme, New York, Nations Unies, série M N83.

Papatheodorou, Andreas (2001), «Why People Travel to Different Places? », Annals of Tourism Research, vol. 28, $\mathrm{n}^{\circ}$ 1, p. 164-179.

Poon, Auliana (1993), Tourism, Technology and Competitive Strategies, Wallingford, CAB International.

Randriamboarison, Rado (2003), La demande touristique en France: Théorie et application, document de travail n'08-A/2003, Groupe d'Étude et de Recherche en Économie Mathématique, Département de sciences économiques et de gestion (GEREM), Université de Perpignan.

Sessa, Alberto (1989), "Characteristics of Tourism », dans Stephen F. Witt et Luiz Moutinho (dir.), Tourism Marketing and Management Handbook, Cambridge, Prentice Hall, p. 354-361.

Stafford, Jean (1996), Microéconomie du Tourisme, Québec, Presses universitaires du Québec. 